\title{
Vacuum Photon Splitting in Lorentz-Violating Quantum Electrodynamics
}

\author{
V. Alan Kostelecký and Austin G.M. Pickering \\ Physics Department, Indiana University, Bloomington, IN 47405 \\ (IUHET 453, December 2002; accepted for publication in Physical Review Letters)
}

\begin{abstract}
Radiative corrections arising from Lorentz violation in the fermion sector induce a nonzero amplitude for vacuum photon splitting. At one loop, the on-shell amplitude acquires both CPTeven and CPT-odd contributions forbidden in conventional electrodynamics.
\end{abstract}

In quantum electrodynamics (QED), a photon traveling in the vacuum has a zero amplitude for decay into multiple on-shell photons at any order in perturbation theory. This classic no-splitting result is a byproduct of Schwinger's work on the proper-time method and the Euler-Heisenberg effective action in QED [1].

Schwinger's result relies heavily on gauge invariance and also implicitly on Lorentz and CPT symmetry. However, Lorentz and CPT symmetry may be broken by effects from the Planck scale [2]. Any effects are expected to be heavily suppressed by at least one power of the Planck mass, which implies most processes in QED and the Standard Model acquire only small Lorentzviolating corrections. Nonetheless, some processes normally excluded may now occur. It is natural to ask how Schwinger's result forbidding on-shell vacuum photon splitting generalizes to the Lorentz-violating case.

The purpose of this work is to discuss the amplitude for vacuum photon splitting in the context of the general Lorentz- and CPT-violating QED extension [3], which includes all possible Lorentz-violating terms involving electron and photon fields. At leading order in certain coefficients for Lorentz violation, we find a nonzero on-shell one-loop amplitude for photon triple splitting.

We work within the renormalizable sector of the general QED extension for a single Dirac field $\psi$ of mass $m$, for which the lagrangian $\mathcal{L}$ is [3,4]

$$
\begin{aligned}
\mathcal{L}= & \frac{1}{2} i \bar{\psi} \Gamma^{\nu} \stackrel{\leftrightarrow}{D_{\nu}} \psi-\bar{\psi} M \psi-\frac{1}{4} F^{\mu \nu} F_{\mu \nu} \\
& -\frac{1}{4}\left(k_{F}\right)_{\kappa \lambda \mu \nu} F^{\kappa \lambda} F^{\mu \nu}+\frac{1}{2}\left(k_{A F}\right)^{\kappa} \epsilon_{\kappa \lambda \mu \nu} A^{\lambda} F^{\mu \nu},
\end{aligned}
$$

where $D_{\mu}$ is the usual covariant derivative and

$$
\begin{aligned}
\Gamma^{\nu} & \equiv \gamma^{\nu}+c^{\mu \nu} \gamma_{\mu}+d^{\mu \nu} \gamma_{5} \gamma_{\mu}+e^{\nu}+i f^{\nu} \gamma_{5}+\frac{1}{2} g^{\lambda \mu \nu} \sigma_{\lambda \mu}, \\
M & \equiv m+a_{\mu} \gamma^{\mu}+b_{\mu} \gamma_{5} \gamma^{\mu}+\frac{1}{2} H_{\mu \nu} \sigma^{\mu \nu} .
\end{aligned}
$$

The Lorentz violation is controlled by the real coefficients $a_{\mu}, b_{\mu}, c_{\mu \nu}, d_{\mu \nu}, e_{\mu}, f_{\mu}, g_{\lambda \mu \nu}, H_{\mu \nu}$ in the fermion sector and $\left(k_{A F}\right)_{\mu},\left(k_{F}\right)_{\kappa \lambda \mu \nu}$ in the photon sector. Inspection of Eq. (1) shows the amplitude for vacuum photon splitting is zero at tree level. The task at hand is thus to investigate possible finite radiative corrections to photon splitting at one loop and at leading order in the coefficients for Lorentz violation. In fact, for our purposes it suffices to restrict attention to a conventional photon sector with negligible $\left(k_{A F}\right)_{\mu},\left(k_{F}\right)_{\kappa \lambda \mu \nu}$. The four coefficients $\left(k_{A F}\right)_{\mu}$ have been strongly constrained using measurements of cosmological birefringence [5-7]. Ten of the
19 independent coefficients in $\left(k_{F}\right)_{\kappa \lambda \mu \nu}$ have also been strongly constrained using spectropolarimetry of cosmological sources, while the other nine can be absorbed into the fermion sector by a field redefinition without loss of generality [8].

With a conventional photon sector, photon splitting in the vacuum is strongly restricted by kinematics. If the incident on-shell photon has energy $E_{1}$ and 3-momentum $\vec{p}_{1}$, and the $n$ photons produced in the decay have energies $E_{j}$ and 3 -momenta $\vec{p}_{j}, j=2,3, \ldots, n+1$, then conservation of 3-momentum implies $\sum_{j}\left|\vec{p}_{j}\right| \geq\left|\vec{p}_{1}\right|$. Since each on-shell photon has 4-momentum $p^{\mu}=(E, \vec{p})$ satisfying $p^{2}=0$ and hence $E=|\vec{p}|$, this inequality is compatible with conservation of energy only if all the $\vec{p}_{j}$ are aligned. The incident photon and the decay products must therefore be collinear. It then follows that the 4-momenta of all photons are mutually orthogonal, $p_{j}^{\mu} p_{k \mu}=0$, and that they are all proportional to some momentum $p_{0}^{\mu}$ satisfying $p_{0}^{2}=0$ on shell. Moreover, the transversality of each physical photon implies that its polarization 4 -vector $\varepsilon^{\mu}$ obeys $p_{\mu} \varepsilon^{\mu}=0$. Together with the requirement of collinearity, this implies $\varepsilon_{j}^{\mu} p_{k \mu}=0$ and that there are at most two linearly independent physical polarization vectors in any process.

In addition to these kinematical constraints, the amplitude for photon splitting must satisfy criteria imposed by symmetry transformations and field redefinitions. First, since the theory (1) is invariant under observer Lorentz transformations, the amplitude must be an observer Lorentz scalar and so any Lorentz indices it contains must be contracted. Second, the properties of the Lorentz-violating operators in Eq. (1) under charge conjugation C imply a generalization of Furry's theorem, which eliminates both divergent and finite contributions arising from certain coefficients [9]. It follows that only $a_{\mu}, d_{\mu \nu}, e_{\mu}, f_{\mu}, H_{\mu \nu}$ can contribute to splitting into an even number of photons, while only $b_{\mu}, c_{\mu \nu}, g_{\lambda \mu \nu}$ can contribute to odd splitting. Third, the behavior of Eq. (1) under parity inversion $\mathrm{P}$ ensures that any contribution to the amplitude linear in $b_{\mu}, d_{\mu \nu}, f_{\mu}$ must come with one factor of the antisymmetric tensor $\epsilon_{\mu \nu \rho \sigma}$. Fourth, gauge invariance demands that the amplitude be invariant when an external photon polarization vector is shifted by an amount proportional to its momentum. The expression for the amplitude must therefore vanish if any one polarization vector is replaced by the corresponding 
external momentum. Finally, in the single-fermion theory (1), some coefficients for Lorentz violation can be removed at linear order by suitable field redefinitions [3] and cannot contribute at leading order to physical processes. So we can ignore potential contributions from $a_{\mu}$, $e_{\mu}, f_{\mu}$, from the antisymmetric parts of $c_{\mu \nu}, d_{\mu \nu}$, and from all but the mixed-symmetry part of $g_{\lambda \mu \nu}$.

The combination of all the above constraints severely restricts the possibilities for photon splitting. For splitting into two photons, we find the only possibility for a nonzero amplitude involves the combination $\varepsilon_{j}^{\mu} H_{\mu \nu} p_{k}^{\nu} \varepsilon_{l}^{\alpha} \varepsilon_{m \alpha}$. For splitting into three photons, the possibilities for a nonzero amplitude include the two combinations $\epsilon_{\mu \nu \rho \sigma} b^{\mu} p^{\nu} \varepsilon_{j}^{\rho} \varepsilon_{k}^{\sigma} \varepsilon_{l}^{\alpha} \varepsilon_{m \alpha}$ and $c_{\mu \nu} p^{\mu} p^{\nu} \varepsilon_{j}^{\alpha} \varepsilon_{k \alpha} \varepsilon_{l}^{\beta} \varepsilon_{m \beta}$. Similar strong constraints can be deduced on the amplitudes for splitting into any number of photons.

To determine whether any of these amplitudes are nonzero, we proceed by direct calculation. For splitting into two photons, the calculation reveals that the overall amplitude vanishes. Photon double splitting is therefore absent, despite the presence of general Lorentz-violating terms in the fermion sector. However, the calculation of photon triple splitting reveals a nonzero amplitude, as we demonstrate next [10].

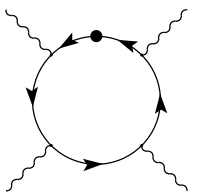

(a)

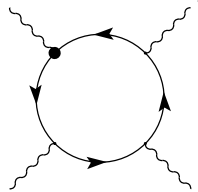

(b)
FIG. 1. Diagrams for one-loop photon triple splitting.

Consider first the $\mathrm{P}$ - and CPT-even contribution to photon triple splitting, which is linear in $c_{\mu \nu}$. This arises from a propagator insertion involving $c_{\mu \nu}$ in the usual one-loop four-point diagram, as shown in Fig. 1a. Note that a vertex insertion of $c_{\mu \nu}$, shown in Fig. 1b, cannot give a gauge-invariant contribution to on-shell photon triple splitting because $c_{\mu \nu}$ always appears contracted with a polarization vector. Denoting the external momenta as $p_{j}, j=1,2,3,4$, and the loop momentum as $k$, and adopting the shorthand notation $k_{1}=k+p_{1}$, $k_{12}=k+p_{1}+p_{2}$, etc., the contribution $G$ from a single graph of the form in Fig. 1a is

$$
G^{\mu_{1} \mu_{2} \mu_{3} \mu_{4}}=\int \frac{d^{4} k}{(2 \pi)^{4}}\left[\frac{\operatorname{Tr}\left[(\not k+m) c^{\mu \nu} \gamma_{\mu} k_{\nu}(\not k+m) \gamma^{\mu_{1}}\left(\not k_{1}+m\right) \gamma^{\mu_{2}}\left(\not k_{12}+m\right) \gamma^{\mu_{3}}\left(\not k_{123}+m\right) \gamma^{\mu_{4}}\right]}{\left(k^{2}-m^{2}\right)^{2}\left(k_{1}^{2}-m^{2}\right)\left(k_{12}^{2}-m^{2}\right)\left(k_{123}^{2}-m^{2}\right)}\right] .
$$

The denominators can be combined in the usual way using Feynman parameters, so that $G=A+B$ with

$$
\begin{aligned}
& A=4 ! \int_{0}^{1} d x_{1} \int_{0}^{1-x_{1}} d x_{2} \int_{0}^{1-x_{12}} d x_{3} \int_{0}^{1-x_{123}} d x_{4} \int \frac{d^{4} k}{(2 \pi)^{4}} 2 k_{\mu} c^{\mu \nu} k_{\nu}\left[\frac{\operatorname{Tr}\left[(\not k+m) \gamma^{\mu_{1}}\left(\not k_{1}+m\right) \gamma^{\mu_{2}}\left(\not k_{12}+m\right) \gamma^{\mu_{3}}\left(\not k_{123}+m\right) \gamma^{\mu_{4}}\right]}{\left(k^{2}-m^{2}+2 k \cdot q\right)^{5}}\right] \\
& B=-3 ! \int_{0}^{1} d x_{1} \int_{0}^{1-x_{1}} d x_{2} \int_{0}^{1-x_{12}} d x_{3} \int \frac{d^{4} k}{(2 \pi)^{4}}\left[\frac{\operatorname{Tr}\left[\left(c^{\mu \nu} \gamma_{\mu} k_{\nu}\right) \gamma^{\mu_{1}}\left(\not k_{1}+m\right) \gamma^{\mu_{2}}\left(\not k_{12}+m\right) \gamma^{\mu_{3}}\left(\not k_{123}+m\right) \gamma^{\mu_{4}}\right]}{\left(k^{2}-m^{2}+2 k \cdot q\right)^{4}}\right],
\end{aligned}
$$

where $x_{12}=x_{1}+x_{2}, x_{123}=x_{1}+x_{2}+x_{3}, q=x_{1} p_{1}+x_{2} p_{12}+x_{3} p_{123}$ with $q^{2}=0$, and the indices on $A, B$ are suppressed for brevity. The trace of an odd number of $\gamma$-matrices vanishes, so the traces can be expanded as polynomials in $m^{2}$ :

$$
A=4 ! \int_{0}^{1} d x_{1} \int_{0}^{1-x_{1}} d x_{2} \int_{0}^{1-x_{12}} d x_{3} \int_{0}^{1-x_{123}} d x_{4}\left(A_{1}+m^{2} A_{2}+m^{4} A_{3}\right), \quad B=3 ! \int_{0}^{1} d x_{1} \int_{0}^{1-x_{1}} d x_{2} \int_{0}^{1-x_{12}} d x_{3}\left(B_{1}+m^{2} B_{2}\right)
$$

where

$$
\begin{aligned}
& A_{1}=\int \frac{d^{4} k}{(2 \pi)^{4}} 2 k_{\mu} c^{\mu \nu} k_{\nu}\left[\frac{\operatorname{Tr}\left[\not k \gamma^{\mu_{1}} \not k_{1} \gamma^{\mu_{2}} \not k_{12} \gamma^{\mu_{3}} \not k_{123} \gamma^{\mu_{4}}\right]}{\left(k^{2}-m^{2}+2 k \cdot q\right)^{5}}\right], \\
& A_{2}=\int \frac{d^{4} k}{(2 \pi)^{4}} 2 k_{\mu} c^{\mu \nu} k_{\nu}\left[\frac{\operatorname{Tr}\left[\not k \gamma^{\mu_{1}} \not k_{1} \gamma^{\mu_{2}} \gamma^{\mu_{3}} \gamma^{\mu_{4}}\right]+\operatorname{Tr}\left[\not k \gamma^{\mu_{1}} \gamma^{\mu_{2}} \not k_{12} \gamma^{\mu_{3}} \gamma^{\mu_{4}}\right]+\operatorname{Tr}\left[\not k \gamma^{\mu_{1}} \gamma^{\mu_{2}} \gamma^{\mu_{3}} \not k_{123} \gamma^{\mu_{4}}\right]}{\left(k^{2}-m^{2}+2 k \cdot q\right)^{5}}\right. \\
& \left.+\frac{\operatorname{Tr}\left[\gamma^{\mu_{1}} \not k_{1} \gamma^{\mu_{2}} \not k_{12} \gamma^{\mu_{3}} \gamma^{\mu_{4}}\right]+\operatorname{Tr}\left[\gamma^{\mu_{1}} \not k_{1} \gamma^{\mu_{2}} \gamma^{\mu_{3}} \not k_{123} \gamma^{\mu_{4}}\right]+\operatorname{Tr}\left[\gamma^{\mu_{1}} \gamma^{\mu_{2}} \not k_{12} \gamma^{\mu_{3}} \not k_{123} \gamma^{\mu_{4}}\right]}{\left(k^{2}-m^{2}+2 k \cdot q\right)^{5}}\right], \\
& A_{3}=\int \frac{d^{4} k}{(2 \pi)^{4}} 2 k_{\mu} c^{\mu \nu} k_{\nu}\left[\frac{\operatorname{Tr}\left[\gamma^{\mu_{1}} \gamma^{\mu_{2}} \gamma^{\mu_{3}} \gamma^{\mu_{4}}\right]}{\left(k^{2}-m^{2}+2 k \cdot q\right)^{5}}\right]
\end{aligned}
$$

and

$$
\begin{aligned}
& B_{1}=-\int \frac{d^{4} k}{(2 \pi)^{4}}\left[\frac{\operatorname{Tr}\left[\gamma^{\mu} c_{\mu \nu} k^{\nu} \gamma^{\mu_{1}} \not k_{1} \gamma^{\mu_{2}} \not k_{12} \gamma^{\mu_{3}} \not k_{123} \gamma^{\mu_{4}}\right]}{\left(k^{2}-m^{2}+2 k \cdot q\right)^{4}}\right] \\
& B_{2}=-\int \frac{d^{4} k}{(2 \pi)^{4}}\left[\frac{\operatorname{Tr}\left[\gamma^{\mu} c_{\mu \nu} k^{\nu} \gamma^{\mu_{1}} \not k_{1} \gamma^{\mu_{2}} \gamma^{\mu_{3}} \gamma^{\mu_{4}}\right]+\operatorname{Tr}\left[\gamma^{\mu} c_{\mu \nu} k^{\nu} \gamma^{\mu_{1}} \gamma^{\mu_{2}} \not k_{12} \gamma^{\mu_{3}} \gamma^{\mu_{4}}\right]+\operatorname{Tr}\left[\gamma^{\mu} c_{\mu \nu} k^{\nu} \gamma^{\mu_{1}} \gamma^{\mu_{2}} \gamma^{\mu_{3}} \not k_{123} \gamma^{\mu_{4}}\right]}{\left(k^{2}-m^{2}+2 k \cdot q\right)^{4}}\right]
\end{aligned}
$$


The traces in these expressions can be reduced using

$$
2 \operatorname{Tr}\left[\gamma_{1} \gamma_{2} \ldots \gamma_{n}\right]=\eta_{12} \operatorname{Tr}\left[\gamma_{3} \gamma_{4} \ldots \gamma_{n}\right]-\eta_{13} \operatorname{Tr}\left[\gamma_{2} \gamma_{4} \ldots \gamma_{n}\right]+\eta_{14} \operatorname{Tr}\left[\gamma_{2} \gamma_{3} \ldots \gamma_{n}\right]-\ldots+\eta_{1 n} \operatorname{Tr}\left[\gamma_{2} \gamma_{3} \ldots \gamma_{n-1}\right]
$$

The full contribution to this vertex from all the relevant graphs, including all permutations over external momenta, must be finite [9]. However, the expression $G$ for a single contributing graph diverges, and the divergences must be isolated by regulation. The integrals can be performed in $d=4-2 \epsilon$ dimensions, using the result

$$
\begin{aligned}
\int \frac{d^{d} k}{(2 \pi)^{4}} \frac{k_{\mu_{1}} \ldots k_{\mu_{n}}}{\left(k^{2}-m^{2}+2 k \cdot q\right)^{\alpha}}=(-1)^{n} \frac{i \pi^{d / 2}\left(-m^{2}\right)^{(d-2 \alpha) / 2}}{\Gamma(\alpha)}\left[\Gamma(\alpha-d / 2) q_{\mu_{1}} \ldots q_{\mu_{n}}\right. \\
+\left(-m^{2} / 2\right) \Gamma(\alpha-1-d / 2)\left(q_{\mu_{1}} \ldots q_{\mu_{(n-2)}} \eta_{\mu_{(n-1)} \mu_{n}}+\right.\text { permutations) } \\
\left.+\ldots+\left(-m^{2} / 2\right)^{n / 2} \Gamma(\alpha-(n+d) / 2) \eta_{\mu_{1} \mu_{2}} \ldots \eta_{\mu_{(n-1)} \mu_{n}}\right] .
\end{aligned}
$$

Since the external momenta are parallel, they can all be written in terms of a single vector $p_{0}^{\mu}$ satisfying $p_{0}^{2}=0$ and $p_{0}^{\mu} \epsilon_{\mu}=0$. We can therefore write $q^{\mu}=q p_{0}^{\mu}$, etc., so that in what follows a quantity such as $q^{2}$ no longer denotes a square of the corresponding four-vector but instead denotes the square of a scalar coefficient. Various terms such as $\not q \gamma^{\mu} \not q$ can then be shown to vanish upon contraction with $\epsilon_{\mu}$, and after some algebra we find that only terms quadratic in $p_{0}^{\mu}$ survive. These terms are finite, so the limit $d \rightarrow 4$ can be taken. The result is:

$$
\begin{array}{r}
A_{1}+m^{2} A_{2}+m^{4} A_{3}=-\frac{i c_{\mu \nu} p_{0}^{\mu} p_{0}^{\nu}}{192 \pi^{2} m^{2}[} \eta^{\mu_{1} \mu_{2}} \eta^{\mu_{3} \mu_{4}}\left(80 q^{2}-q\left(44 p_{1}+32 p_{2}+12 p_{3}\right)+4 p_{1} p_{123}\right) \\
+\eta^{\mu_{1} \mu_{3}} \eta^{\mu_{2} \mu_{4}}\left(-112 q^{2}+q\left(60 p_{1}+40 p_{2}+20 p_{3}\right)-4 p_{1} p_{123}\right) \\
\left.+\eta^{\mu_{1} \mu_{4}} \eta^{\mu_{2} \mu_{3}}\left(80 q^{2}-q\left(52 p_{1}+32 p_{2}+20 p_{3}\right)+4 p_{1} p_{123}\right)\right]
\end{array}
$$

Inserting these expressions into Eq. (5), we can explicitly integrate over the Feynman parameters since these appear at most quadratically in the integrands. The result for $G=A+B$ is:

$$
\begin{gathered}
G^{\mu_{1} \mu_{2} \mu_{3} \mu_{4}}=-\frac{i c_{\mu \nu} p_{0}^{\mu} p_{0}^{\nu}}{1440 \pi^{2} m^{2}}\left[\eta^{\mu_{1} \mu_{2}} \eta^{\mu_{3} \mu_{4}}\left(102 p_{1}^{2}+24 p_{2}^{2}+37 p_{3}^{2}+89 p_{1} p_{2}+115 p_{1} p_{3}+81 p_{2} p_{3}\right)\right. \\
-\eta^{\mu_{1} \mu_{3}} \eta^{\mu_{2} \mu_{4}}\left(126 p_{1}^{2}+48 p_{2}^{2}+41 p_{3}^{2}+133 p_{1} p_{2}+119 p_{1} p_{3}+93 p_{2} p_{3}\right) \\
\left.+\eta^{\mu_{1} \mu_{4}} \eta^{\mu_{2} \mu_{3}}\left(48 p_{1}^{2}+37 p_{3}^{2}+11 p_{1} p_{2}+85 p_{1} p_{3}+63 p_{2} p_{3}\right)\right]
\end{gathered}
$$

The full amplitude for the photon triple splitting at this order is obtained by summing the contributions from 24 graphs. These consist of four diagrams of the form in Fig. 1a with $c_{\mu \nu}$ insertions on the different fermion propagators, with three distinct topological channels for each diagram, and two orientations of the fermion loop for each channel. All these contributions can be derived from the result (11) for $G$ by suitable index permutations. Defining $P$ as $P\left(f\left(\mu_{1}, \mu_{2}, \mu_{3}, \mu_{4}, p_{1}, p_{2}, p_{3}, p_{4}\right)\right) \equiv f\left(\mu_{4}, \mu_{1}, \mu_{2}, \mu_{3}, p_{4}, p_{1}, p_{2}, p_{3}\right)$, then the sum of the four $c_{\mu \nu}$ insertions is given by $R=G+P(G)+P(P(G))+P(P(P(G)))$ for one channel. To obtain different topological channels, we interchange first $\mu_{1}$ and $\mu_{2}$ and subsequently $\mu_{1}$ and $\mu_{4}$ without changing the momentum indices. Hence, the full contribution to the photon triple splitting amplitude $T_{c}$ is $T_{c}=2\left(R+C_{12}(R)+C_{14}(R)\right)$, where $C_{12}\left(f\left(\mu_{1}, \mu_{2}, \mu_{3}, \mu_{4}\right)\right)=f\left(\mu_{2}, \mu_{1}, \mu_{3}, \mu_{4}\right)$, $C_{14}\left(f\left(\mu_{1}, \mu_{2}, \mu_{3}, \mu_{4}\right)\right)=f\left(\mu_{4}, \mu_{2}, \mu_{3}, \mu_{1}\right)$. Explicitly, we find

$$
\begin{array}{r}
T_{c}^{\mu_{1} \mu_{2} \mu_{3} \mu_{4}}=-\frac{i c_{\mu \nu} p_{0}^{\mu} p_{0}^{\nu}}{60 \pi^{2} m^{2}}\left[\eta^{\mu_{1} \mu_{2}} \eta^{\mu_{3} \mu_{4}}\left(9 p_{1}^{2}-8 p_{2}^{2}-p_{3}^{2}+2 p_{1} p_{2}+16 p_{1} p_{3}-18 p_{2} p_{3}\right)\right. \\
+\eta^{\mu_{1} \mu_{3}} \eta^{\mu_{2} \mu_{4}}\left(4 p_{1}^{2}-8 p_{2}^{2}+4 p_{3}^{2}-8 p_{1} p_{2}+16 p_{1} p_{3}-8 p_{2} p_{3}\right) \\
\left.+\eta^{\mu_{1} \mu_{4}} \eta^{\mu_{2} \mu_{3}}\left(-p_{1}^{2}-8 p_{2}^{2}+9 p_{3}^{2}-18 p_{1} p_{2}+16 p_{1} p_{3}+2 p_{2} p_{3}\right)\right]
\end{array}
$$

where we have used $p_{1}+p_{2}+p_{3}+p_{4}=0$. Thus, a nonzero coefficient $c_{\mu \nu}$ yields a finite CPT-even contribution $T_{c}$ to the amplitude for photon triple splitting, even in the collinear limit. 
By a similar set of calculations we obtain a finite CPT-odd contribution $T_{b}$ to the amplitude for photon triple splitting from a nonzero coefficient $b_{\mu}$, again in the collinear limit:

$$
T_{b}^{\mu_{1} \mu_{2} \mu_{3} \mu_{4}}=\frac{b_{\mu} p_{0 \nu}}{6 \pi^{2} m^{2}}\left[\left(9 p_{1}+3 p_{3}\right) \eta^{\mu_{1} \mu_{2}} \epsilon^{\mu_{3} \mu_{4} \mu \nu}-\left(5 p_{1}+4 p_{2}+3 p_{3}\right) \eta^{\mu_{1} \mu_{3}} \epsilon^{\mu_{2} \mu_{4} \mu \nu}-\left(3 p_{1}+p_{3}\right) \eta^{\mu_{2} \mu_{3}} \epsilon^{\mu_{1} \mu_{4} \mu \nu}\right] .
$$

Note that these results also imply nonzero contributions in the off-shell, non-collinear case. However, the existence of nonzero amplitudes in the collinear limit suffices to show that Schwinger's no-splitting result is vitiated in the presence of Lorentz violation [11].

The nonzero amplitudes (12), (13) must arise from gauge-invariant terms in the effective action $S_{\text {eff }}$ for the Lorentz-violating QED extension (1). The form of $S_{\text {eff }}$ is not known explicitly, and it would be of interest to obtain it. The amplitudes (12), (13) are determined in the collinear limit and therefore cannot be used to deduce uniquely the terms in $S_{\text {eff }}$ from which they arise. However, $S_{\text {eff }}$ could include expressions such as $c_{\mu \nu} F^{\mu \kappa} F_{\kappa}{ }^{\nu} \partial^{-2} F^{\alpha \beta} F_{\alpha \beta}$, which for transverse photons in the collinear limit generates terms of the structure necessary to reproduce the result (12). Note that the appearance of the inverse box operator is to be expected in the effective action of a massless field [12].

We close with brief remarks about an issue that is of some interest but lies beyond our present scope: the determination of a physical rate for on-shell photon triple splitting. For a massive particle, the decay rate is defined in the rest frame with a kinematic factor inversely proportional to the mass, so cannot trivially be extended to the massless case. Since the theory (1) is observer Lorentz covariant, it may be possible to define a physically consistent decay rate in terms of the particle energy in the observer frame [13]. There is also a separate kinematical issue to consider because collinear momenta occupy a set of measure zero in the phase space. However, a physical analogy is provided by the collinear parametric down conversion of photons in optically active crystals, for which photon multiple splitting has been experimentally observed [14]. The Lorentz violation induces an analogous effective optical activity of the vacuum [3,8]. Equations (12) and (13) imply any nonzero decay rate would be quadratically suppressed in the small coefficients $c_{\mu \nu}$ and $b_{\mu}[15]$. Nonetheless, Lorentz-violating photon degradation over cosmic distances might lead to observable effects on the redshift $[16,17]$, conceivably even affecting open issues such as the origin of the cosmological constant.

We thank R. Jackiw for discussions. This work is supported in part by the United States Department of Energy under grant DE-FG02-91ER40661.

[1] J.S. Schwinger, Phys. Rev. 82, 664 (1951).

[2] V.A. Kostelecký and S. Samuel, Phys. Rev. D 39, 683 (1989); 40, 1886 (1989); Phys. Rev. Lett. 63, 224 (1989);
V.A. Kostelecký and R. Potting, Nucl. Phys. B 359, 545 (1991); Phys. Lett. B 381, 89 (1996).

[3] V.A. Kostelecký and R. Potting, Phys. Rev. D 51, 3923 (1995); D. Colladay and V.A. Kostelecký, Phys. Rev. D 55, 6760 (1997); 58, 116002 (1998).

[4] Nonrenormalizable Lorentz-violating terms generate suppressed contributions to the effective action at low energies; see V.A. Kostelecký and R. Lehnert, Phys. Rev. D 63, 065008 (2001). Although outside our present scope, it would be of interest to consider photon splitting in various nonrenormalizable subsets of the general Lorentzviolating QED. These include noncommutative QED; see S.M. Carroll et al., Phys. Rev. Lett. 87, 141601 (2001); Z. Guralnik et al., Phys. Lett. B 517, 450 (2001); C.E. Carlson et al., Phys. Lett. B 518, 201 (2001).

[5] S.M. Carroll, G. Field, and R. Jackiw, Phys. Rev. D 41, 1231 (1990).

[6] Radiative corrections to $\left(k_{A F}\right)_{\mu}$ are considered in $\mathrm{R}$. Jackiw and V.A. Kostelecký, Phys. Rev. Lett. 82, 3572 (1999) and references therein.

[7] Photon decay with all coefficients zero other than $\left(k_{A F}\right)_{\mu}$ has recently been studied by C. Adam and F.R. Klinkhamer, Nucl. Phys. B 657, 214 (2003).

[8] V.A. Kostelecký and M. Mewes, Phys. Rev. Lett. 87, 251304 (2001); Phys. Rev. D 66, 056005 (2002).

[9] V.A. Kostelecky, C.D. Lane and A.G.M. Pickering, Phys. Rev. D 65, 056006 (2002).

[10] The methods adopted here can also be applied to verify directly the vanishing of the amplitude for photon triple splitting in conventional QED.

[11] O.W. Greenberg, Phys. Rev. Lett. 89, 231602 (2002) has shown that CPT violation must be accompanied by Lorentz violation, so the latter can be viewed as the primary effect even for $T_{b}$.

[12] For instance, in the two-dimensional Schwinger model for QED, the one-loop effective action is proportional to $F_{\mu \nu} \partial^{-2} F^{\mu \nu}$. See J. Schwinger, Phys. Rev. 128, 2425 (1962). An example in gauge theory can be found in R. Jackiw and S.Y. Pi, Phys. Lett. B 368, 131 (1996).

[13] P. Havas, Am. J. Phys. 34, 753 (1966).

[14] J.A. Giordmaine and R.C. Miller, Phys. Rev. Lett. 14, 973 (1965).

[15] In contrast, non-collinear decays are quartically suppressed. See T. Jacobson, S. Liberati, and D. Mattingly, hep-ph/0209264, and Ref. [7].

[16] For example, dimensional analysis suggests photons with $E \sim m$ have Hubble-scale lifetime if $c_{\mu \nu}$ is of order $10^{-19}$, an experimentally viable Planck-scale suppression.

[17] Historically, photon splitting in conventional Lorentzinvariant QED was suggested as a possible explanation of the red shift: P. Roman, Magyar Fiz. Foly 3, 115 (1955). A phenomenological treatment of cosmological limits is given by D.C. Wilkins, Phys. Rev. D 21, 2122 (1980). 